CERN-PH-TH/2008-071

MCTP-08-13

KIAS-P08025

NSF-KITP-08-18

\title{
Higgs Boson Exempt No-Scale Supersymmetry with a Neutrino Seesaw: Implications for Lepton Flavor Violation and Leptogenesis
}

\author{
Eung Jin Chun ${ }^{a}$, Jason L. Evans ${ }^{b}$, David E. Morrissey ${ }^{b, d}$, James D. Wells ${ }^{c, b, d}$ \\ a Korean Institute for Advanced Study (KIAS) \\ Hoegiro 87, Dongdaemun-gu Seoul 130-722, Korea \\ ${ }^{b}$ Michigan Center for Theoretical Physics (MCTP) \\ University of Michigan, Ann Arbor, MI 48109 \\ c CERN, Theory Division, CH-1211 Geneva 23, Switzerland \\ ${ }^{d}$ Kavli Institute for Theoretical Physics \\ University of California, Santa Barbara, CA 93106-4030
}

June 16, 2021

\begin{abstract}
Motivated by the observation of neutrino oscillations, we extend the Higgs boson exempt no-scale supersymmetry model (HENS) by adding three heavy right-handed neutrino chiral supermultiplets to generate the light neutrino masses and mixings. The neutrino Yukawa couplings can induce new lepton flavor violating couplings among the soft terms in the course of renormalization group running down from the boundary scale. We study the effects this has on the predictions for low-energy probes of lepton flavor violation (LFV). Heavy right-handed neutrinos also provide a way to generate the baryon asymmetry through leptogenesis. We find that consistency with LFV and leptogenesis puts strong requirements on either the form of the Yukawa mass matrix or the smallness of the Higgs up soft mass. In all cases, we generically expect that new physics LFV is non-zero and can be found in a future experiment.
\end{abstract}




\section{Contents}

1 Introduction $\quad 2$

2 LFV in the HENS Model with Heavy Neutrinos 4

2.1 Off-Diagonal HENS Soft Terms from RG Running . . . . . . . . . . . . . 5

2.2 HENS LFV . . . . . . . . . . . . . . . . . . . . . 6

2.3 Constraints on the HENS Model from LFV . . . . . . . . . . . . . . . . 6

3 Leptogenesis in $\nu$ HENS $\quad 12$

4 HENS Leptogenesis with LFV Constraints 14

4.1 Simultaneous Constraints . . . . . . . . . . . . . . 15

4.2 Neutrino Yukawa Matrix Structures . . . . . . . . . . . . . . . 16

5 Conclusions $\quad 18$

\section{Introduction}

Supersymmetry (SUSY) is a well-motivated and elegant possibility for new physics beyond the Standard Model (SM). However, SUSY can only be an approximate symmetry of nature. The requirement of (soft) SUSY breaking introduces many new unconstrained parameters to the theory that can be phenomenologically problematic. For example, generic soft supersymmetry breaking couplings of $\mathrm{TeV}$ size would lead to excessive amounts of flavor mixing and CP violation [1, 2].

A simple way to address this flavor-mixing problem of low-energy supersymmetry is to arrange for all the matter scalar soft terms to vanish at a common input scale $M_{c}$. Provided this input scale is well above the electroweak scale, acceptably large scalar soft terms will be regenerated in the course of renormalization group running from the high scale to the scale of the soft supersymmetry breaking couplings [3, 4, 5, 6, 7]. Since the scalar soft terms generated in this way come mostly from loops of gauginos, they are nearly flavor universal and therefore consistent with the current bounds on flavor mixing.

This scenario for addressing the SUSY flavor problem is realized within Higgs-exempt noscale supersymmetry (HENS) [8]. In this model, the squark and slepton soft terms all vanish at a high input scale, taken to be the scale of unification $M_{G U T} \simeq 2 \times 10^{16} \mathrm{GeV}$, while the gaugino masses are non-zero there. This can be achieved within an extra-dimensional setup as in gaugino mediation [4, 5, 6], or by nearly conformal running [7, 9, 10, 11]. However, unlike pure gaugino mediation and traditional no-scale models, the Higgs scalar squared masses are 
allowed to be non-vanishing at the input scale $M_{G U T}$. With this small modification, that does not contribute appreciably to flavor mixing, it is possible to obtain a cosmologically-favored neutralino LSP [8]. Under the assumption of gaugino universality, the free parameters of the HENS model at the input scale $M_{G U T}$ are

$$
\tan \beta, m_{H_{u}}^{2}, m_{H_{d}}^{2}, M_{1 / 2}, \operatorname{sgn}(\mu)
$$

where $M_{1 / 2}$ is the universal gaugino mass. With this small number of inputs, the HENS model is able to account for the dark matter, can be made consistent with all current experimental bounds, and leads to exciting collider phenomenology [8].

While the HENS model is phenomenologically enticing, it cannot explain the observation of neutrino oscillations [12]. This shortcoming can be resolved by supplementing the model with three heavy singlet right-handed neutrino chiral superfields with the superpotential couplings

$$
W=W_{0}+N Y_{\nu} L H_{u}+\frac{1}{2} N M_{N} N
$$

where $W_{0}$ is the MSSM superpotential, $N$ are the right-handed neutrinos, $M_{N}$ is their Majorana mass matrix, and $Y_{\nu}$ is the neutrino Yukawa matrix. By taking the singlet neutrino masses $M_{N_{i}}$ to be much larger than the electroweak scale, very small masses can be generated for the left-handed neutrinos by the seesaw mechanism [12]. Integrating out the heavy neutrino states yields the effective superpotential coupling

$$
W_{e f f}=W_{0}-\frac{1}{2}\left(Y_{\nu}^{T} M_{N}^{-1} Y_{\nu}\right)_{i j}\left(L_{i} H_{u}\right)\left(L_{j} H_{u}\right)
$$

For $M_{N} \sim 10^{12} \mathrm{GeV}$, this interaction can generate correct light neutrino masses at the weak scale with the neutrino Yukawa couplings on the order of unity, $Y_{\nu} \sim 0.1$.

Adding heavy right-handed neutrinos to the HENS scenario also introduces a new flavormixing problem to the model. In running the soft parameters in the full theory (Eq. (2) from the input scale $M_{G U T}$ down to the heavy singlet neutrino scale $M_{N}$, the neutrino Yukawa couplings generate non-universal contributions to the soft masses for the charged leptons [13]. Such couplings are dangerous because they are a source of lepton flavor violation (LFV) [13], for which the experimental bounds are extremely strong. This in turn imposes stringent constraints on the heavy neutrino sector.

Although adding right-handed neutrinos to SUSY models can lead to problematic LFV rates, such extensions also have some attractive collateral features. One of these is the possibility of generating the baryon asymmetry via leptogenesis [14, 15]. Heavy right-handed neutrinos provide all the necessary ingredients for baryogenesis. Lepton number is not a conserved quantity in the neutrino sector since the Majorana masses of the heavy righthanded neutrinos violate lepton number $L$ by two units. Combined with the $(B+L)$-violation due to $S U(2)_{L}$ sphaleron transitions in the early universe [16, 17], there exists a source of baryon number violation. The neutrino sector also provides a new source of $\mathrm{CP}$ violation from the complex neutrino Yukawa matrix. This $\mathrm{CP}$ violation can manifest itself in the outof-equilibrium decays and scatterings of the right-handed neutrinos in the early universe. 
Together, these features fulfill the three Sakharov conditions for baryogenesis [18], which can be realized through the mechanism of leptogenesis.

Requiring that the neutrino-extended HENS ( $\nu$ HENS) model account for the baryon asymmetry of the universe while respecting the current bounds on LFV leads to constraints on the structure of the neutrino Yukawa matrix and the right-handed neutrino masses. Previous studies combining the requirements for leptogenesis with the bounds from LFV can be found in Refs. [19, 20, 21, 22]. Compared to these previous works, we study the constraints from LFV within the context of a specific model for which the lack of flavor mixing outside the neutrino sector is well-motivated. An interesting result along these lines is that the amount of LFV in the HENS model is largely controlled by the value of $m_{H_{u}}^{2}$ at the high input scale. The degree to which the neutrino sector parameters are constrained therefore depends strongly on the size of $m_{H_{u}}^{2}$.

The outline of this paper is as follows. In Section 2 we investigate LFV in the HENS model induced by the inclusion of heavy right-handed neutrinos. Using the current bounds on LFV processes, we obtain constraints on the underlying model. In Section 3 we investigate whether it is possible for the HENS model with right-handed neutrinos to account for the baryon asymmetry by way of thermal leptogenesis. We combine the results of Sections 2 and 3 in Section 4, where we examine whether leptogenesis can generate the baryon asymmetry while satisfying bounds from LFV. Finally, Section 5 is reserved for our conclusions.

\section{LFV in the HENS Model with Heavy Neutrinos}

We begin by considering the constraints on the HENS model from LFV induced by the inclusion of heavy right-handed neutrinos. These constraints depend strongly on the parameters in the neutrino sector such as the Majorana masses for the right-handed neutrinos and the neutrino Yukawa matrix. Some of these neutrino sector parameters have been determined by the measurements of the light neutrino mass differences and mixings [23, 24]. In anticipation of computing the LFV constraints, we collect here our notation and assumptions about the neutrino sector.

In terms of the couplings in the full superpotential of Eq. (2), the low-energy effective superpotential of Eq. (3) implies that the light neutrino mass matrix is given by

$$
m_{\nu_{i j}}=\frac{v_{u}^{2}}{2}\left(Y_{\nu}^{T} M_{N}^{-1} Y_{\nu}\right)_{i j}
$$

This matrix can be diagonalized by the unitary PMNS matrix $U$ [25, 26]. Following the standard convention, we will parameterize the PMNS matrix with three real angles and three phases according to

$$
U=\mathcal{O}_{23}\left(\theta_{23}\right) \Gamma_{\delta} \mathcal{O}_{13}\left(\theta_{13}\right) \Gamma_{\delta}^{*} \mathcal{O}_{12}\left(\theta_{12}\right) \times \operatorname{diag}\left[e^{i \alpha_{1} / 2}, e^{i \alpha_{2} / 2}, 1\right]
$$

where $\Gamma_{\delta}=\operatorname{diag}\left(1,1, e^{i \delta}\right)$, and $\mathcal{O}_{i j}=\left[\left(c_{i j}, s_{i j}\right),\left(-s_{i j}, c_{i j}\right)\right]$ with $c_{i j}=\cos \theta_{i j}$ and $s_{i j}=\sin \theta_{i j}$. 
It is convenient to make use of the known structure of the light neutrino mass matrix to parameterize the neutrino Yukawa matrix $Y_{\nu}$ according to [27]

$$
Y_{\nu}=\frac{1}{v_{u}} \sqrt{M_{N}} R \sqrt{m_{\nu_{\text {diag }}}} U^{\dagger}
$$

where $R$ is a complex orthogonal matrix, $M_{N}$ is the diagonal right-handed neutrino mass matrix, and $m_{\nu_{\text {diag }}}$ is the diagonalized left-handed neutrino mass matrix. Here, and throughout this paper, we will always work in a field basis such that the right-handed neutrino and charged lepton mass matrices are diagonal. Since the $R$ matrix is complex orthogonal, we can parameterize it in terms of three complex angles according to

$$
R=\operatorname{diag}( \pm 1, \pm 1, \pm 1) \mathcal{O}_{12}\left(\theta_{12 R}\right) \mathcal{O}_{23}\left(\theta_{23 R}\right) \mathcal{O}_{31}\left(\theta_{31 R}\right)
$$

with $\mathcal{O}_{i j}=\left[\left(c_{i j R}, s_{i j R}\right),\left(-s_{i j R}, c_{i j R}\right)\right]$, where $c_{i j R}=\cos \theta_{i j R}$ and $s_{i j R}=\sin \theta_{i j R}$. Note that since these angles are complex, the components of $R$ are not bounded in magnitude. This means that some of the entries in the neutrino Yukawa matrix could be quite large, but through cancellations among the see-saw contributions, still give rise to acceptably small light neutrino masses. In order to avoid too much fine-tuning in this regard, we will only consider $R$ matrices with $\left|R_{i j}\right|<10$, which corresponds roughly to a tuning of less than $10 \%$ in the light neutrino mass matrix. Our choices for the light neutrino masses and mixings are listed in Appendix A.

\subsection{Off-Diagonal HENS Soft Terms from RG Running}

Without heavy right-handed neutrinos, the HENS model is safe in terms of lepton-flavor violation $(\mathrm{LFV})$. With heavy right-handed neutrinos, lepton-flavor violating couplings can arise among the scalar soft terms in the course of renormalization group (RG) running down from the input scale $M_{G U T}$. The strict experimental limits on LFV will in turn lead to constraints on the neutrino Yukawa couplings and right-handed neutrino masses. Since this new source of FCNC in the HENS model arises from RG running, and not the SUSY breaking mechanism, its amplitude will have a similar form to that found in mSUGRA models.

The dominant contribution to the off-diagonal flavor-mixing components of the scalar soft squared masses is well-approximated by keeping only the leading logarithmic term in the RG running. ${ }^{1}$ With this approximation applied to the boundary conditions appropriate to the HENS model $\left(m_{\tilde{f}}^{2}=0, m_{H_{u}}^{2}, m_{H_{d}}^{2} \neq 0\right)$, we obtain [13, 29]

$$
m_{\tilde{L}_{i \neq j}}^{2}=-\frac{1}{8 \pi^{2}} m_{H u}^{2} \sum_{k} Y_{\nu k i}^{*} Y_{\nu k j} \ln \left(\frac{M_{G U T}}{M_{N_{k}}}\right) .
$$

To this order of approximation, the flavor non-diagonal elements in the scalar trilinear soft couplings and the right-handed slepton soft masses vanish. When the constraints on the neutrino Yukawa couplings from LFV are applied, the corrections to the diagonal components of the scalar masses are numerically very small; less than about $5 \mathrm{GeV}$ in most of the parameter space. However, when these corrections could be relevant we have included them.

\footnotetext{
1 The leading-log approximation breaks down for $M_{1 / 2} \gtrsim 1000 \mathrm{GeV}$ and $\left|m_{H_{u}}^{2}\right| \lesssim(100 \mathrm{GeV})^{2}[28$. To avoid this, we include subleading terms in our numerical analysis.
} 


\subsection{HENS LFV}

The off-diagonal soft terms introduced by RG running, given in Eq. (8), will induce LFV transitions of the type $\ell_{i} \rightarrow \ell_{j} \gamma$. The leading contributions to the branching fractions for these transitions can be written as [29, 30, 31]

$$
B\left(\ell_{i} \rightarrow \ell_{j} \gamma\right)=\frac{\alpha}{4 \Gamma\left(\ell_{i}\right)} m_{\ell_{i}}^{5}\left|A_{L}^{(i j)}\right|^{2}
$$

where $\Gamma\left(\ell_{i}\right)$ is the total decay width of lepton $\ell_{i}$, and the amplitude $A_{L}^{(i j)}$ has the schematic form 29,30

$$
A_{L}^{(i j)}=m_{\tilde{L}_{i \neq j}}^{2} F_{L}^{(i j)}
$$

with $F_{L}^{(i j)}$ a combination of loop functions that depend on the chargino, neutralino, and slepton masses. These loop functions are such that the dominant contribution to $B\left(\ell_{i} \rightarrow \ell_{j} \gamma\right)$ scales approximately as $m_{\tilde{L}_{i \neq j}}^{2} \tan ^{2} \beta M_{1 / 2}^{-8}$. Note also that in this leading contribution to the LFV branching fractions, the flavor violating term $m_{\tilde{L}_{i \neq j}}^{2}$ can be factored out. This will allow us to discuss the effects of the neutrino sector and the supersymmetry breaking sector separately.

The differences in the branching fractions of Eq. (9) for the HENS model compared to mSUGRA lie in the form $m_{\tilde{L}_{i \neq j}}^{2}$ and the low-scale sparticle masses. However, $m_{\tilde{L}_{i \neq j}}^{2}$ is qualitatively similar in the two theories and will be of the same order of magnitude for both theories as long as $m_{H_{u}}^{2} \sim m_{0}^{2}+a_{0}^{2}$. The loop functions $F_{L}^{(i j)}$ are also qualitatively similar, but differ in the masses of the gauginos and sleptons running in the loops that appear as their arguments. From this, there can be a slight enhancement of the LFV rates in HENS relative to mSUGRA because the slepton masses tend to be somewhat lighter in the HENS model. On the other hand, the LFV rates can be reduced in the HENS model relative to mSUGRA by arranging for $m_{H_{u}}^{2}$ to vanish, which suppresses the leading source of lepton flavor mixing given in Eq. (8). As shown in Ref. 8], it is often possible to obtain a consistent phenomenology with $m_{H_{u}}^{2} \sim 0$, especially for $\tan \beta \lesssim 30$. To obtain a similar suppression in mSUGRA, one would need both $m_{0}$ and $a_{0}$ to be quite small which can be phenomenologically problematic [32, 33].

\subsection{Constraints on the HENS Model from LFV}

The possibility of inducing LFV places significant constraints on right-handed neutrino extensions of the HENS model. The two strongest bounds on new sources of LFV come from searches for $\mu \rightarrow e \gamma$ and $\tau \rightarrow \mu \gamma$ transitions:

$$
\begin{aligned}
B(\mu \rightarrow e \gamma) & <1.2 \times 10^{-11}, \\
B(\tau \rightarrow \mu \gamma) & <4.5 \times 10^{-8},
\end{aligned}
$$




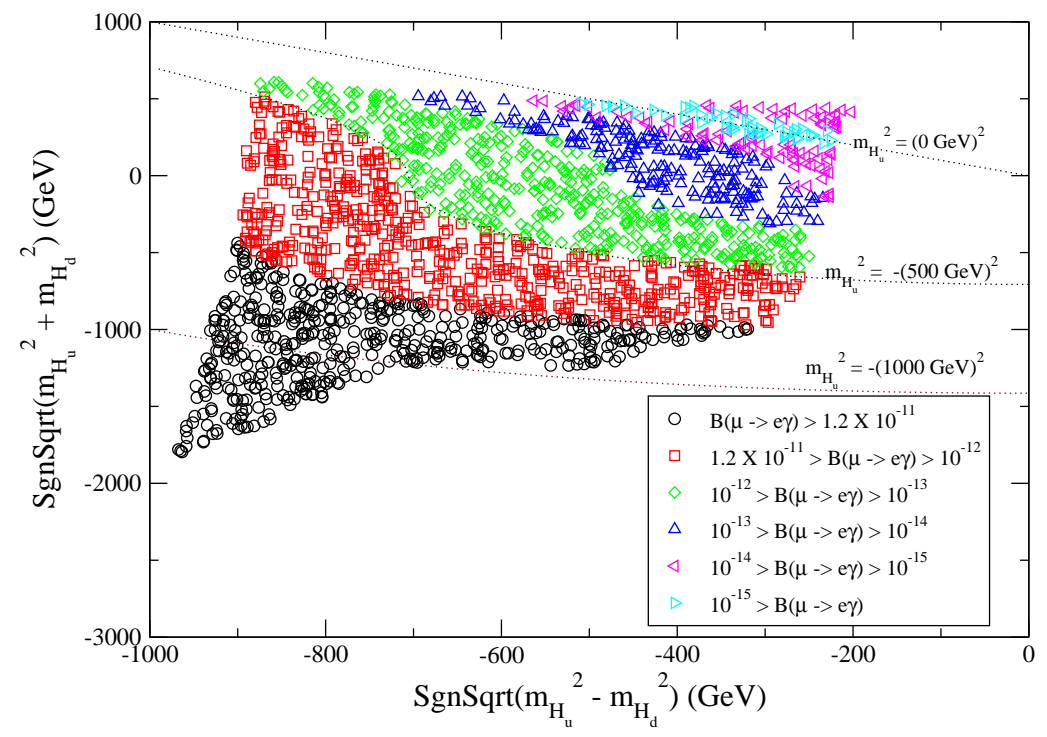

Figure 1: $B(\mu \rightarrow e \gamma)$ as a function of the HENS model parameters $m_{H_{u}}^{2}$ and $m_{H_{d}}^{2}$ at the high input scale. The other model parameters are $M_{1 / 2}=300 \mathrm{GeV}, \tan \beta=10$, and $\operatorname{sgn}(\mu)=1$ as well as neutrino-sector parameters $\theta_{12 R}=\theta_{13 R}=\theta_{23 R}=\pi / 4+i \ln (\sqrt{2}), M_{N_{3}}=10^{12} \mathrm{GeV}$, $M_{N_{2}}=10^{11} \mathrm{GeV}$, and $M_{N_{1}}=10^{10} \mathrm{GeV}$. All points in this plot are consistent with collider phenomenology constraints and have a neutralino LSP.

It was shown in Ref. [29] that if these bounds are satisfied, the bounds on other experimentally searched-for channels such as $B(\mu \rightarrow 3 e)$ will generally be satisfied as well.

In Fig. 1 we show the dependence of the LFV branching fraction $B(\mu \rightarrow e \gamma)$ on the high-scale input values of $m_{H_{u}}^{2}$ and $m_{H_{d}}^{2}$ in the HENS model with right-handed neutrinos. The other HENS parameters are taken to be $M_{1 / 2}=300 \mathrm{GeV}, \tan \beta=10$, and $\operatorname{sgn}(\mu)=1$. This value of $M_{1 / 2}$ is about as small as is possible in the HENS model while still obtaining a sufficiently heavy Higgs boson [8]. The points in this figure cover the region of the HENS parameter space that is consistent with all collider and phenomenological constraints other than from LFV, and that has a neutralino LSP. The neutrino-sector parameters are taken to be $M_{N_{3}}=10^{12} \mathrm{GeV}, M_{N_{2}}=10^{11} \mathrm{GeV}, M_{N_{1}}=10^{10} \mathrm{GeV}$, the light neutrino masses are as described in Appendix A with $m_{3}=0.05 \mathrm{eV}$, and the $R$-matrix angles (see Eq. (7)) are equal to $\theta_{12 R}=\theta_{13 R}=\theta_{23 R}=\pi / 4+i \ln (\sqrt{2})$. These particular values of the neutrino sector parameters were chosen for convenience, but we have checked that they lead to typical amounts of LFV. The decreasing trend in $B(\mu \rightarrow e \gamma)$ from bottom-left to top-right in this figure corresponds largely to a decreasing value of $m_{H_{u}}^{2}$. This is not surprising given Eq. (8), which shows that the leading contribution to lepton flavor mixing is proportional to $m_{H_{u}}^{2}$.

Fig. 2 shows the dependence of the LFV branching fraction $B(\mu \rightarrow e \gamma)$ on $m_{H_{u}}^{2}$ and $m_{H_{d}}^{2}$ 


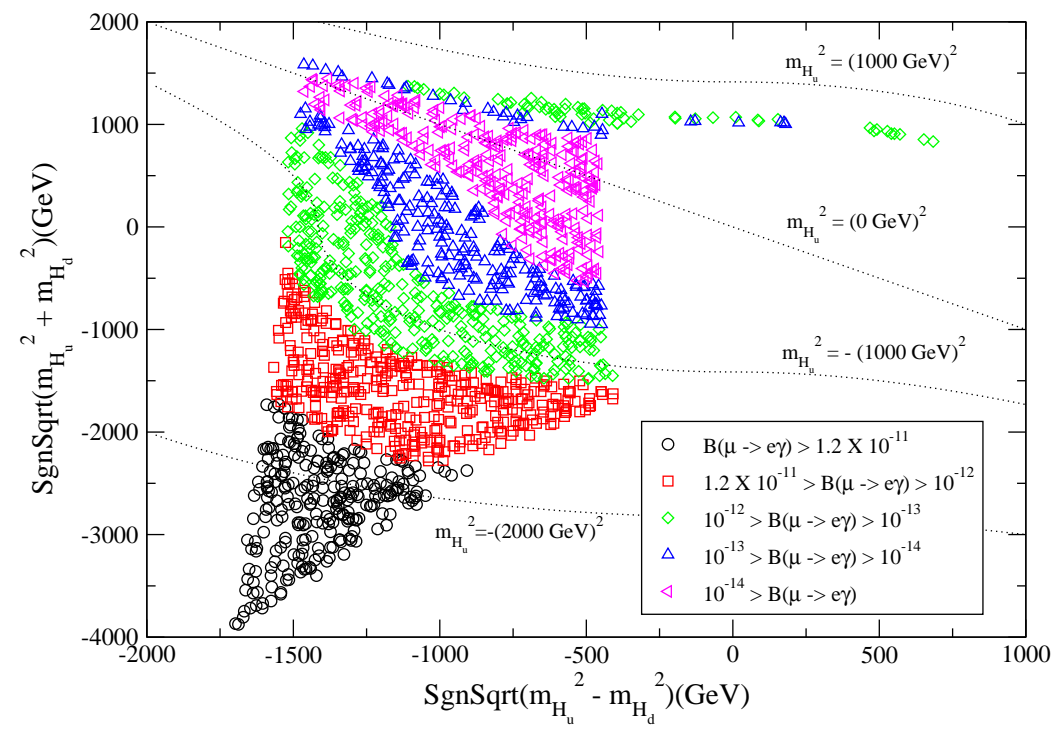

Figure 2: $\quad B(\mu \rightarrow e \gamma)$ as a function of the HENS model parameters $m_{H_{u}}^{2}$ and $m_{H_{d}}^{2}$. The other model parameters are $M_{1 / 2}=500 \mathrm{GeV}$ and $\tan \beta=10$, as well as neutrino-sector parameters $\theta_{12 R}=\theta_{13 R}=\theta_{23 R}=\pi / 4+i \ln (\sqrt{2}), M_{N_{3}}=10^{12} \mathrm{GeV}, M_{N_{2}}=10^{11} \mathrm{GeV}$, and $M_{N_{1}}=10^{10}$. All points in this plot are consistent with collider phenomenology constraints and have a neutralino LSP.

for the same neutrino sector parameters as Fig. 1 , but now with $M_{1 / 2}=500 \mathrm{GeV}$. Also as before, $\tan \beta=10, \operatorname{sgn}(\mu)=1$, and all points shown are consistent with collider constraints and have a neutralino LSP. Compared to Fig. 1, the LFV rates are considerably lower. This can be understood in terms of the general scaling of all the superpartner masses with $M_{1 / 2}$, and the fact that larger superpartner masses suppress the loop functions appearing in Eq. (10). Aside from this scaling, the shapes of the contours in the two figures are very similar, with the dominant variation in the branching fraction due to the changing input value of $m_{H_{u}}^{2}$.

In Fig. 3 we illustrate the dependence of the LFV branching ratio $B(\mu \rightarrow e \gamma)$ on $m_{H_{u}}^{2}$ and $m_{H_{d}}^{2}$ for $\tan \beta=30, M_{1 / 2}=500 \mathrm{GeV}$, and $\operatorname{sgn}(\mu)=1$ over the allowed parameter space in the HENS model. All points in the plot satisfy collider phenomenology constraints and have a neutralino LSP. The values of the neutrino sector parameters are the same as in Figs. 1 . and 2. The variation of $B(\mu \rightarrow e \gamma)$ in this plot again tracks the value of $m_{H_{u}}^{2}$. However, the overall values of the LFV branching ratio $B(\mu \rightarrow e \gamma)$ are larger than in the previous figures. There are two reasons for this. The first is that the expression for $B(\mu \rightarrow e \gamma)$ scales like $\tan ^{2} \beta$. The second reason for the relative enhancement in the LFV rates is that larger values of $\tan \beta$ also enhance the $\tau$ Yukawa coupling, making it more likely to obtain a stau LSP. To obtain a neutralino LSP, which we demand as a phenomenological constraint, $m_{H_{u}}^{2}$ 


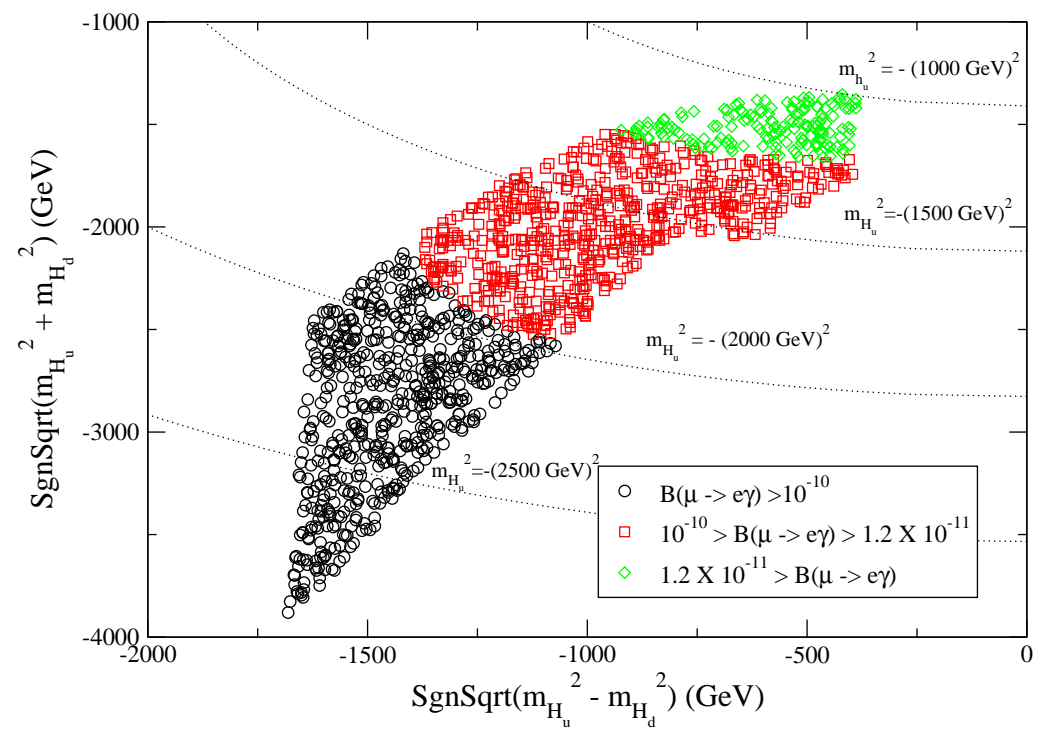

Figure 3: $\quad B(\mu \rightarrow e \gamma)$ as a function of the HENS model parameters $m_{H_{u}}^{2}$ and $m_{H_{d}}^{2}$. The other model parameters are $M_{1 / 2}=500 \mathrm{GeV}$ and $\tan \beta=30$, as well as neutrino-sector parameters $\theta_{12 R}=\theta_{13 R}=\theta_{23 R}=\pi / 4+i \ln (\sqrt{2}), M_{N_{3}}=10^{12} \mathrm{GeV}, M_{N_{2}}=10^{11} \mathrm{GeV}$, and $M_{N_{1}}=10^{10}$. All points in this plot are consistent with collider phenomenology constraints and have a neutralino LSP.

must be large in magnitude and negative in sign. This limits the suppression of $B(\mu \rightarrow e \gamma)$ that occurs in the HENS model as $m_{H_{u}}^{2}$ becomes small. With these two sources of relative enhancement at larger values of $\tan \beta$, we see that in the present example there are very few parameter points consistent with the bound on $B(\mu \rightarrow e \gamma)$ listed in Eq. (11).

In the plots discussed above, the LFV rates depend most sensitively on the parameter $m_{H_{u}}^{2}$. To better illustrate this relationship, we plot in Fig. 4 the same sets of points as in Figs. 1, 2, and 3 in terms of $B(\mu \rightarrow e \gamma)$ as a function of $m_{H_{u}}^{2}$. These sets correspond to $\tan \beta=10$ and $M_{1 / 2}=300 \mathrm{GeV}, \tan \beta=10$ and $M_{1 / 2}=500 \mathrm{GeV}$, and $\tan \beta=30$ and $M_{1 / 2}=500 \mathrm{GeV}$ respectively, with $m_{H_{d}}^{2}$ scanned over. The values of the neutrino sector parameters are the same as in the previous plots. As expected from Eq. (8), the LFV rates drop precipitously as $m_{H_{u}}^{2} \rightarrow 0$. When this occurs, only the much smaller terms beyond the leading order term given in Eq. (8) contribute to lepton flavor mixing. These subleading terms scale like $M_{1 / 2}$, and can not be zeroed out due to the phenomenological lower bounds on $M_{1 / 2}$. Fig. 4 also illustrates the scaling of $B(\mu \rightarrow e \gamma)$ with $M_{1 / 2}$, which we expect to go like $M_{1 / 2}^{-8}$, as well as the enhancement of the LFV rates for larger values of $\tan \beta$. There is a dip in the branching fraction at $m_{H_{u}}^{2} \simeq(700)^{2} \mathrm{GeV}^{2}$. This corresponds to $M_{1} \simeq \mu$, leading to a large mixing among the neutralinos and a cancellation between contributions to the amplitude. 


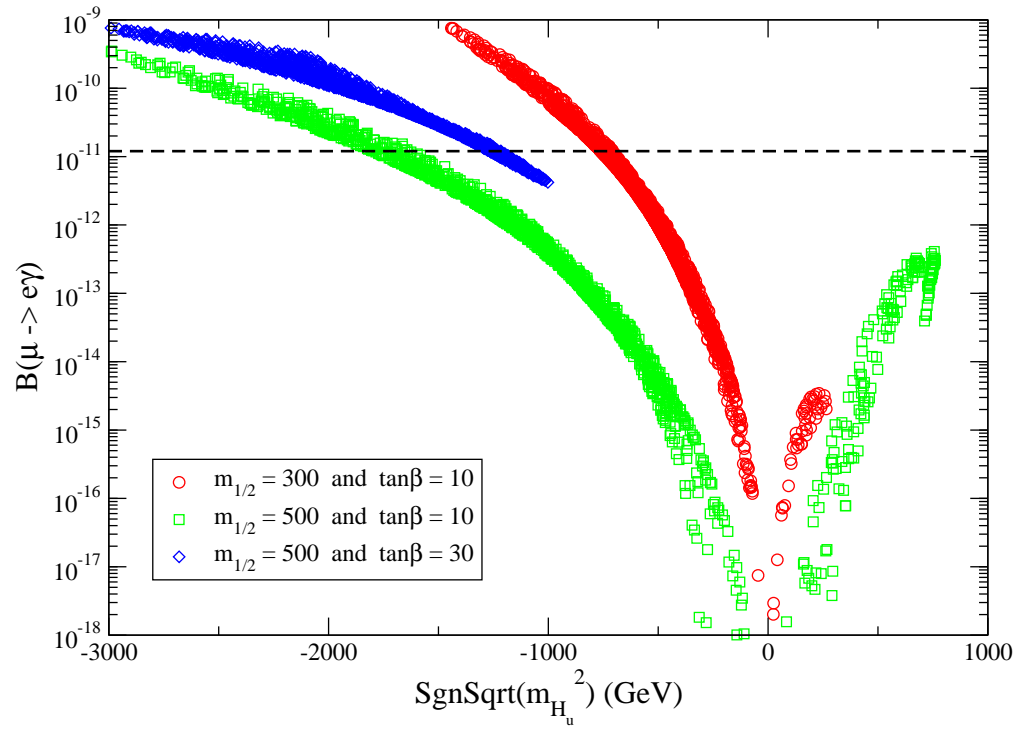

Figure 4: $\quad B(\mu \rightarrow e \gamma)$ as a function of $m_{H_{u}}^{2}$ at the high input scale for several values of $M_{1 / 2}$ and $\tan \beta$. Values of $m_{H_{d}}^{2}$ were scanned over, and all points are consistent with collider phenomenology constraints and have a neutralino LSP. The neutrino sector parameters are given by $\theta_{12 R}=\theta_{13 R}=\theta_{23 R}=\pi / 4+i \ln (\sqrt{2}), M_{N_{3}}=10^{12} \mathrm{GeV}, M_{N_{2}}=10^{11} \mathrm{GeV}$, and $M_{N_{1}}=10^{10} \mathrm{GeV}$. The dashed line in this figure corresponds to the experimental LFV bound $B(\mu \rightarrow e \gamma)<1.2 \times 10^{-11}$.

We have concentrated so far on the specific branching fraction $B(\mu \rightarrow e \gamma)$. The related branching fractions $B(\tau \rightarrow \mu \gamma)$ and $B(\tau \rightarrow e \gamma)$ both have a very similar dependence on the HENS model parameters. Plots of these branching fractions as a function of $m_{H_{u}}^{2}$ are nearly identical in both shape and overall normalization to those in Fig. 4. However, since the experimental upper bounds on the branching fractions of these $\tau$ modes are more than a couple of orders of magnitude larger than the $\mu$ mode, they provide much weaker constraints on the neutrino-enhanced HENS parameter space. We will therefore concentrate most strongly on the $\mu \rightarrow e \gamma$ mode in the present work.

Having studied the dependence of the LFV rates on the HENS model parameters for a particular (but typical) set of neutrino sector parameters, let us next examine the dependence of the LFV rates on the details of the neutrino sector. In Fig. 5 we show the branching fraction $B(\mu \rightarrow e \gamma)$ as a function of the heaviest right-handed neutrino mass $M_{N_{3}}$. Of the heavy neutrino masses, this one usually plays the most important role in determining the amount of LFV. The HENS model parameters for this plot are $\tan \beta=10, M_{1 / 2}=300 \mathrm{GeV}$, $m_{H_{u}}^{2}=-(511 \mathrm{GeV})^{2}$ and $m_{H_{d}}^{2}=-(668 \mathrm{GeV})^{2}$. These values produce a phenomenologically consistent spectrum, which we list in Appendix B, and are not unusual in terms of LFV. The light neutrino masses are as described in Appendix A. The remaining neutrino sector 


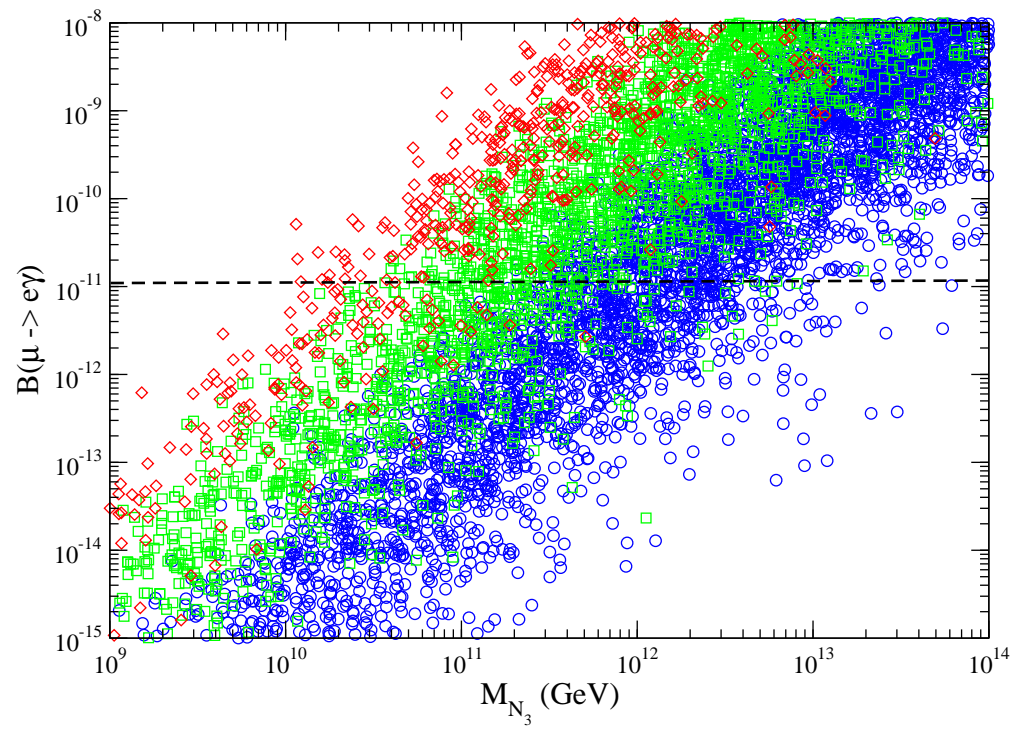

Figure 5: $B(\mu \rightarrow e \gamma)$ as a function of the heaviest right-handed neutrino mass $M_{N_{3}}$ for the HENS parameters $m_{H_{u}}^{2}=-(668)^{2} \mathrm{GeV}^{2}, m_{H_{d}}^{2}=-(511)^{2} \mathrm{GeV}^{2}, \tan \beta=10$, and $M_{1 / 2}=$ $300 \mathrm{GeV}$. The blue circles, green squares, and red diamonds correspond to $\operatorname{Max}\{|R|\}<2$, $2<\operatorname{Max}\{|R|\}<5$, and $5<\operatorname{Max}\{|R|\}<10$, respectively. The dashed line represents the experimental bound of $B(\mu \rightarrow e \gamma)<1.2 \times 10^{-11}$.

parameters were scanned over: heavy neutrino masses lie in the range $M_{N} \in\left[10^{7}, 10^{14}\right] \mathrm{GeV}$ with no particular hierarchy between them, and the $R$ matrix angles range over $\operatorname{Re}(\theta) \in$ $[0,2 \pi]$ and $\operatorname{Im}(\theta) \in[-2,2]$. Within the plot, the blue circles, green squares, and red diamonds correspond to $\operatorname{Max}\{|R|\} \in[0,2], \operatorname{Max}\{|R|\} \in[2,5]$, and $\operatorname{Max}\{|R|\} \in[5,10]$. Recall that since $R$ is a complex orthogonal matrix, its components are unbounded, although large components require a fine-tuning to obtain small neutrino masses.

The two most important neutrino sector quantities for $B(\mu \rightarrow e \gamma)$ are the structure of the $R$ matrix and the value of $M_{N_{3}}$. The importance of both quantities can be seen in Fig. 5 . In general, smaller neutrino Yukawa couplings lead to less lepton flavor mixing. Thus, given Eq. (6), it is not surprising that smaller components in the $R$ matrix, and lower values of $M_{N_{3}}$ lead to lower values of $B(\mu \rightarrow e \gamma)$. What is more interesting is the wide range of values of this branching fraction for a given fixed value of $M_{N_{3}}$. This indicates that certain textures of the neutrino Yukawa matrix can greatly reduce the amount of LFV. On account of these various sensitivities, it is difficult to demarcate a region of parameter space consistent with the LFV bounds other than by what we have illustrated in Fig. 5. Certain challenging sets of neutrino sector parameters require $M_{N_{3}}<10^{10} \mathrm{GeV}$, while for other neutrino parameters the requirement can be weakened to $M_{N_{3}}<10^{13} \mathrm{GeV}$. More concrete constraints can be derived in certain limits, such as when the right-handed neutrinos are strongly hierarchical. 


\section{Leptogenesis in $\nu$ HENS}

The primary motivation for heavy right-handed neutrinos is to explain the findings of neutrino oscillation experiments. However, heavy neutrinos also provide a mechanism to account for the baryon asymmetry, which is measured to be [38]

$$
Y_{B}=\frac{n_{B}-n_{\bar{B}}}{s}=(8.7 \pm 0.3) \times 10^{-11} .
$$

With heavy right-handed neutrinos, this baryon asymmetry can be generated through the process of leptogenesis [14, 15]. As the universe cools, the heavy neutrinos fall out of equilibrium and decay. If there is a significant amount of CP violation in the neutrino sector, these decays can induce a net lepton number. This lepton number is subsequently reprocessed into a net baryon asymmetry through the $(B+L)$-violating sphaleron transitions [17]. In the present section we investigate whether the HENS model with heavy right-handed neutrinos can explain the baryon asymmetry through thermal leptogenesis.

We use the results of Ref. [39] to compute the baryon density due to thermal leptogenesis in the HENS model. In particular, we take into account flavor effects [39, 40, 41, 42, 43] arising from interactions of the charged Yukawa couplings. Motivated both by the apparent hierarchy of light neutrino masses and the desire to reduce the amount of washout of the lepton asymmetry generated by heavy neutrino decays, we will focus on mildly hierarchical right-handed neutrino masses, with $M_{N_{1}}<M_{N_{2,3}} / 3$.

The baryon asymmetry due to thermal leptogenesis can be expressed in terms of the CP and $\mathrm{L}$ asymmetry $\epsilon_{\alpha}$ and the effective neutrino mass $m_{\alpha}$ for each flavor $\alpha=e, \mu, \tau$, and the washout parameter $\eta$. In the hierarchical limit of $M_{N_{1}} \ll M_{N_{2,3}}, \epsilon_{\alpha}$ and $m_{\alpha}$ are given by [39]

$$
\begin{aligned}
\epsilon_{\alpha} & \simeq-\frac{3 M_{N_{1}}}{16 \pi v_{u}^{2}} \frac{\operatorname{Im}\left[\sum_{i, j} m_{i}^{1 / 2} m_{j}^{3 / 2} U_{\alpha i}^{*} U_{\alpha j} R_{1 i} R_{1 j}\right]}{\sum_{k} m_{k}\left|R_{1 k}\right|^{2}} \\
\tilde{m}_{\alpha} & \equiv \frac{\left|Y_{\nu 1 \alpha}\right|^{2} v_{u}^{2}}{M_{N_{1}}}=\left|\sum_{k} R_{1 k} m_{k}^{1 / 2} U_{\alpha k}^{*}\right|^{2} .
\end{aligned}
$$

A simple approximate form for the washout parameter $\eta$ is [39]

$$
\eta\left(\widetilde{m}_{\alpha}\right)=\left[\left(\frac{\widetilde{m}_{\alpha}}{8.25 \times 10^{-3} \mathrm{eV}}\right)^{-1}+\left(\frac{0.2 \times 10^{-3} \mathrm{eV}}{\widetilde{m}_{\alpha}}\right)^{-1.16}\right]^{-1} .
$$

The two terms in this expression interpolate between the weak (first term) and strong (second term) washout regimes. The first term in Eq. (17), corresponding to weak washout, assumes there is no initial abundance (thermal or otherwise) of right-handed neutrinos, and that the only sources of right-handed neutrinos are inverse decays and scattering. This is a conservative assumption, as the effective value of $\eta$ can be enhanced if the initial state has a non-vanishing heavy neutrino density.

For right-handed neutrino masses in the range $\left(1+\tan ^{2} \beta\right) 10^{9} \mathrm{GeV} \lesssim M_{N_{1}} \lesssim(1+$ $\left.\tan ^{2} \beta\right) 10^{12} \mathrm{GeV}$ the two lepton flavor approximation is appropriate, and the resulting baryon 
density is [39]

$$
Y_{B} \simeq-\frac{10}{31 g_{*}}\left[\epsilon_{2} \eta\left(\frac{541}{761} \widetilde{m}_{2}\right)+\epsilon_{\tau} \eta\left(\frac{494}{761} \widetilde{m}_{\tau}\right)\right] .
$$

In this expression, $g_{*}$ is the usual number of relativistic degrees of freedom, $\widetilde{m}_{2} \equiv \widetilde{m}_{e}+\widetilde{m}_{\mu}$ and $\epsilon_{2} \equiv \epsilon_{e}+\epsilon_{\mu}$. For lighter right-handed neutrino states, with mass in the range $(1+$ $\left.\tan ^{2} \beta\right) 10^{5} \mathrm{GeV} \lesssim M_{N_{1}} \lesssim\left(1+\tan ^{2} \beta\right) 10^{9} \mathrm{GeV}$, we must account for all three lepton flavors. The appropriate expression for the baryon asymmetry in this case is [39]

$$
Y_{B} \simeq-\frac{10}{31 g_{*}}\left[\epsilon_{e} \eta\left(\frac{93}{110} \widetilde{m}_{e}\right)+\epsilon_{\mu} \eta\left(\frac{19}{30} \widetilde{m}_{\mu}\right)+\epsilon_{\tau} \eta\left(\frac{19}{30} \widetilde{m}_{\tau}\right)\right]
$$

In Fig. 6 we show the baryon density due to leptogenesis in the HENS model with heavy right-handed neutrinos as a function of the lightest heavy neutrino mass $M_{N_{1}}$. The HENS model parameters are set to $M_{1 / 2}=300 \mathrm{GeV}, \tan \beta=10, m_{H_{u}}^{2}=-(668 \mathrm{GeV})^{2}$, and $m_{H_{d}}^{2}=-(511 \mathrm{GeV})^{2}$. The superpartner mass spectrum for these values is listed in Appendix B, and is phenomenologically acceptable aside from LFV constraints. We expect these parameters to be typical in terms of leptogenesis. The neutrino sector parameters were scanned over, with the blue circles, green squares, and red diamonds corresponding to $\operatorname{Max}\{|R|\}<2,2<\operatorname{Max}\{|R|\}<5$, and $5<\operatorname{Max}\{|R|\}<10$, respectively.

Fig. 6 indicates that there is a lower bound on $M_{N_{1}}$ if thermal leptogenesis is to be the source of the baryon asymmetry of the universe. The minimal value of $M_{N_{1}}$ that works is on the order of $10^{10} \mathrm{GeV}$, which is consistent with the results of Refs. [44, 45, 46]. This plot also shows that the final baryon asymmetry is reduced as the magnitudes of the entries in the $R$ matrix become larger. The reason for this is that larger values of $\left|R_{i j}\right|$ enhance $\tilde{m}_{\alpha}$, which increases the amount of washout. In the strong washout regime, which we find to be the case throughout much of the parameter space, the lepton asymmetry produced in right-handed neutrino decays is thereby greatly diluted. To obtain a sufficiently large lepton asymmetry to explain the baryon excess in this regime, $M_{N_{1}}$ must be larger than about $10^{10} \mathrm{GeV}$. This can cause difficulties for avoiding the experimental constraints on LFV, as we will discuss later.

Let us also make note of the fact that the lower bound on $M_{N_{1}}$ of about $10^{10} \mathrm{GeV}$ suggests that the reheating temperature after inflation was larger than this if thermal leptogenesis is to explain the baryon asymmetry. In supersymmetric models, such large reheating temperatures lead to the overproduction of gravitinos [47]. Within the HENS model with an input scale on the order of $M_{G U T}$ and an underlying gravity or gaugino mediation of supersymmetry breaking, we expect gravitino masses on the order of the weak scale [48]. Gravitinos of this mass decay during nucleosynthesis, and can ruin the ratios of the light element abundances for $T_{R H} \gtrsim 10^{7 \pm 1} \mathrm{GeV}$ [49]. A couple of possible approaches to this problem are resonant enhancements of the lepton asymmetry as the heavy neutrinos become nearly degenerate that allow $M_{N_{1}}$ to be lowered further [50, 51, 52, 53, 54], or the non-thermal production of heavy right-handed neutrinos after inflation [55, 56]. 


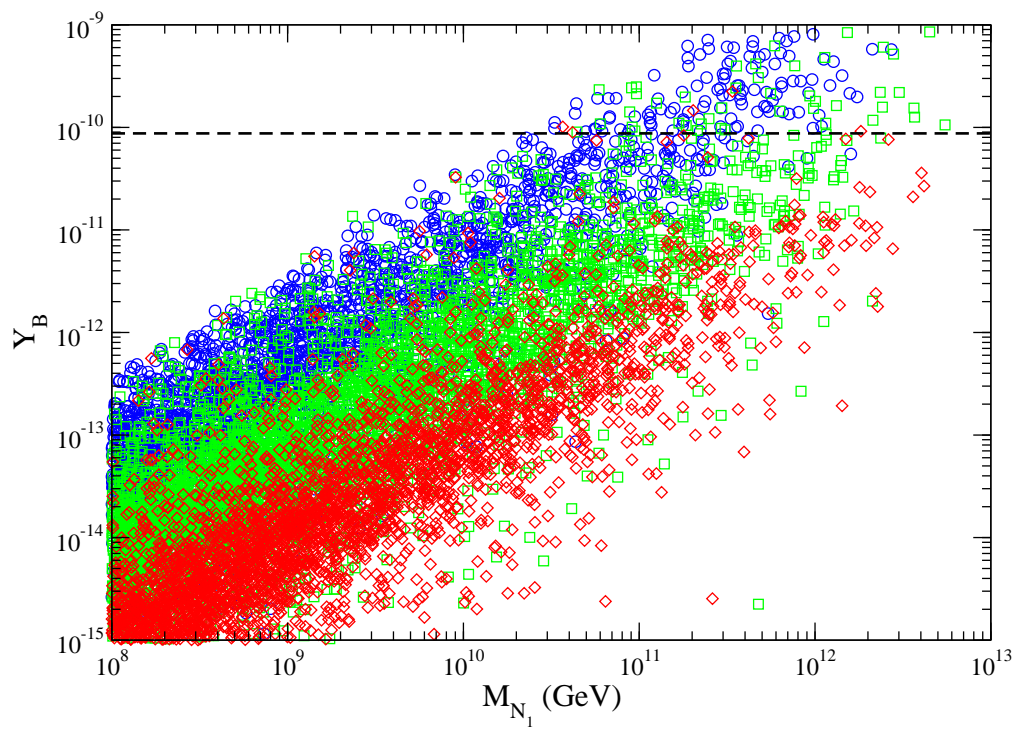

Figure 6: Baryon density due to leptogenesis in the HENS model as a function of $M_{N_{1}}$. The HENS model parameter were set to $m_{H_{u}}^{2}=-(668)^{2} \mathrm{GeV}^{2}, m_{H_{d}}^{2}=-(511)^{2} \mathrm{GeV}^{2}, \tan \beta=10$, and $M_{1 / 2}=300 \mathrm{GeV}$, and the neutrino sector parameters were scanned over. The blue circles, green squares, and red diamonds correspond to $\operatorname{Max}\{|R|\}<2,2<\operatorname{Max}\{|R|\}<5$, and $5<\operatorname{Max}\{|R|\}<10$, respectively. The dashed line represents the measured baryon density $Y_{B}=(8.7 \pm 0.3) \times 10^{-11}$.

\section{HENS Leptogenesis with LFV Constraints}

In the previous two sections we have examined the LFV constraints on the HENS model with heavy right-handed neutrinos, and we have investigated whether this model can account for the baryon asymmetry through thermal leptogenesis. In the present section, we combine these considerations, and study whether the HENS model can be successful in both regards at the same time. To be concrete, we focus on two particular points in the HENS parameter space. For these points, we study many different structures of the neutrino sector, with the one simplifying assumption of slightly hierarchical right-handed neutrino masses with $M_{N_{1}} \lesssim M_{N_{2,3}} / 3$

We will refer to the two HENS model parameter sets as points A and B. Both points have $\tan \beta=10, M_{1 / 2}=300 \mathrm{GeV}$, and $\operatorname{sgn}(\mu)>0$. For point A, the Higgs sector parameters at the input scale are $m_{H_{u}}^{2}=-(668)^{2} \mathrm{GeV}^{2}$ and $m_{H_{d}}^{2}=-(511)^{2} \mathrm{GeV}^{2}$. The corresponding input values for point $\mathrm{B}$ are $m_{H_{u}}^{2}=-(100)^{2} \mathrm{GeV}^{2}, m_{H_{d}}^{2}=-(359)^{2} \mathrm{GeV}^{2}$. The resulting low-energy spectra for these two points are phenomenologically consistent, aside from LFV constraints. We list their mass spectra in Appendix B. The crucial difference between the 


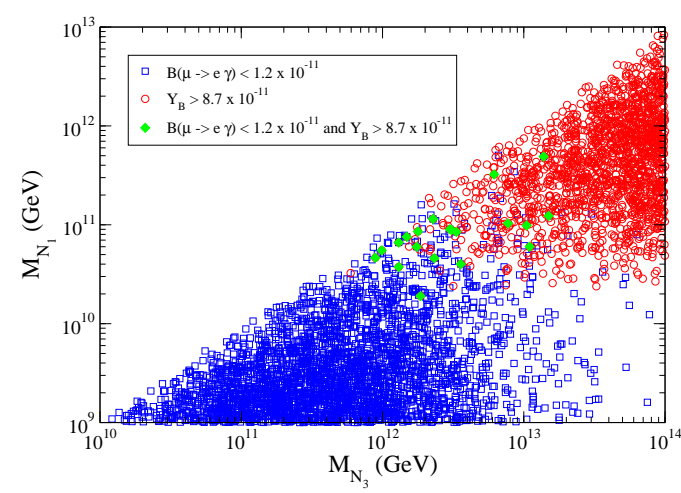

(a)

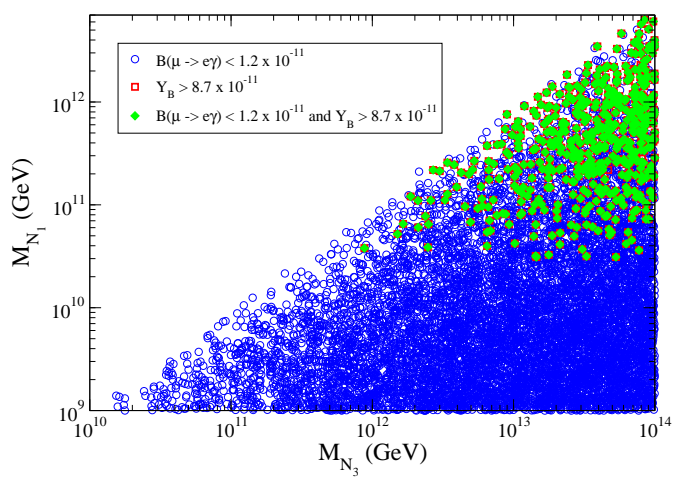

(b)

Figure 7: HENS parameter points in the $M_{N_{3}}-M_{N_{1}}$ plane consistent with LFV constraints (blue squares), baryogenesis through thermal leptogenesis (red circles), or both simultaneously (green diamonds). The panel on the left (a) is for HENS parameter set A, with $M_{1 / 2}=300 \mathrm{GeV}, \tan \beta=10, m_{H_{u}}^{2}=-(668)^{2} \mathrm{GeV}^{2}$, and $m_{H_{d}}^{2}=-(511)^{2} \mathrm{GeV}^{2}$. The panel on the right (b) is for HENS parameter set $\mathrm{B}$, with $M_{1 / 2}=300 \mathrm{GeV}, \tan \beta=10$, $m_{H_{u}}^{2}=-(100)^{2} \mathrm{GeV}^{2}$, and $m_{H_{d}}^{2}=-(359)^{2} \mathrm{GeV}^{2}$. In both plots we have scanned over neutrino sector parameters.

two parameter points is that the input value of $m_{H_{u}}^{2}$ is much larger for point $\mathrm{A}$ than for point B.

\subsection{Simultaneous Constraints}

In Section 2 we found that LFV constraints favor smaller values of $M_{N_{3}}$. On the other hand, in Section 3 we found that thermal leptogenesis prefers larger values of $M_{N_{1}}<M_{N_{3}}$. The tension between these two requirements is illustrated in Fig. 7, where we plot points in the $M_{N_{3}}-M_{N_{1}}$ plane that are consistent with LFV constraints, that generate enough of a baryon asymmetry through thermal leptogenesis, or that satisfy both conditions. The left-hand panel of this figure corresponds to point A described above, while the right-hand panel corresponds to point B. In both panels, we have scanned over heavy neutrino masses $M_{N_{i}}$, as well as the light neutrino masses and the values of the $U$ and $R$ mixing matrices subject to the constraints listed in Appendix A. The blue squares in the figure are points that obey the LFV constraints, the red circles are points that generate enough of a baryon excess, and the green diamonds satisfy both requirements.

Only a very small subset of the points in Fig. 7 for set A are consistent with both the

LFV constraints and leptogenesis. This is primarily the result of the large value of $m_{H_{u}}^{2}$ for this parameter set, which leads to large LFV rates unless $M_{N_{3}}$ is very small. This in turn pushes down the possible range of values of $M_{N_{1}}$, making leptogenesis less effective. Only for 
a small and special subset of the neutrino sector parameters can both requirements be met. We will discuss these requirements in more detail below. In contrast, there are many points for parameter set B for which both the LFV and leptogenesis constraints are met. Indeed, very few of the points that are consistent with generating the baryon asymmetry through leptogenesis do not satisfy the LFV constraints. This is due to the LFV constraints being very weak given the relatively small value of $m_{H_{u}}^{2}$ for this parameter set.

\subsection{Neutrino Yukawa Matrix Structures}

We found above that only a small subset of the neutrino sector parameters allowed for the HENS parameter set A to be consistent with the constraints from LFV while generating the baryon asymmetry via thermal leptogenesis. The combination of these two requirements selects a particular structure for the neutrino Yukawa matrix which we describe here. Due to the assumed hierarchy among the right-handed neutrinos, the Yukawa matrix will generally decrease in size from row three to row one. Thus, the leading contributions to the off-diagonal components of $m_{L_{i j}}^{2}$ responsible for LFV are typically

$$
m_{\tilde{L}_{i j}}^{2}=-\frac{m_{H_{u}}^{2}}{8 \pi^{2}}\left(Y_{\nu 3 i}^{*} Y_{\nu 3 j} t_{3}+Y_{\nu 2 i}^{*} Y_{\nu 2 j} t_{2}\right)
$$

where $t_{i}=\ln \left(M_{G U T} / M_{N_{i}}\right)$. This feature selects out the $Y_{\nu 3 i}$ and $Y_{\nu 2 i}$ components of the neutrino Yukawa matrix as being particularly important.

In Fig. 8 we show the dependence of the LFV branching fraction $B(\mu \rightarrow e \gamma)$ on the $Y_{\nu 3 i}$ and $Y_{\nu 2 i}$ components of the neutrino Yukawa matrix for the HENS model parameter set $\mathrm{A}$ described above and in Appendix B. The points in this plot correspond to different values of the $R$ and $U$ matrix elements, and (hierarchical) right-handed neutrino masses. With the spectrum of parameter set $\mathrm{A}$, the $\mu \rightarrow e \gamma$ branching fraction can be written as

$$
B(\mu \rightarrow e \gamma)=(1400 \mathrm{GeV})^{-4}\left|m_{\tilde{L}_{21}}^{2}\right|^{2}
$$

With $M_{N_{3}}=10^{11} \mathrm{GeV}$, for example, this translates into a constraint on the Yukawa couplings of

$$
Y_{\nu 32}^{*} Y_{\nu 31}+Y_{\nu 22}^{*} Y_{\nu 21} \frac{t_{2}}{t_{3}}<9.6 \times 10^{-5}
$$

where $t_{i}=\ln \left(M_{G U T} / M_{N_{i}}\right)$. This constraint can be met in two different ways: both $\left|Y_{\nu 32}\right|\left|Y_{\nu 31}\right|$ and $\left|Y_{\nu 22}\right|\left|Y_{\nu 21}\right|$ can be separately very small, or $Y_{\nu 32}^{*} Y_{\nu 31}$ and $Y_{\nu 22}^{*} Y_{\nu 21}$ can cancel against each other. It is this cancellation that leads to the pointed structure in Fig. 8.

The constraints on the neutrino Yukawa couplings become even stronger when we demand successful leptogenesis as well. In Fig. 9 we show the equivalent plot to Fig. 8 for HENS parameter set A, but now restricted to points that are consistent with thermal leptogenesis. Clearly, larger values of the Yukawa couplings are required for successful leptogenesis. For these points to also be consistent with LFV constraints, there must be a significant cancellation between $Y_{\nu 32}^{*} Y_{\nu 31}$ and $Y_{\nu 22}^{*} Y_{\nu 21}$ to suppress $B(\mu \rightarrow e \gamma)$, as suggested by Eq. (22). With the present sensitivities, the bounds on LFV in $\tau$ decays do not significantly constrain 


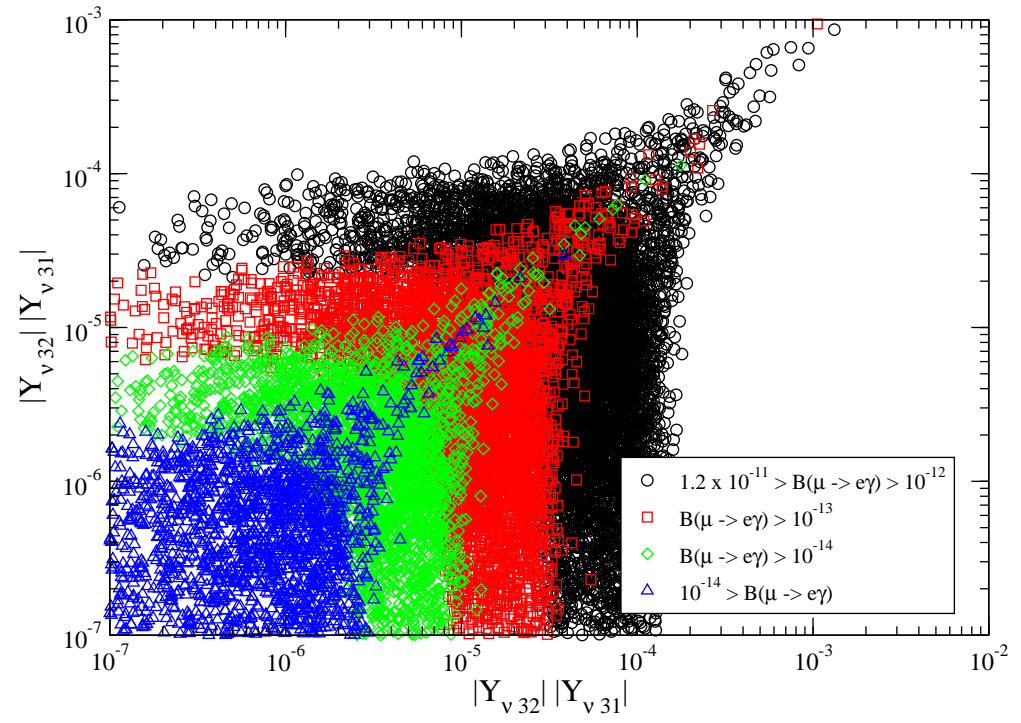

Figure 8: $B(\mu \rightarrow e \gamma)$ in the plane of $\left|Y_{\nu 32}\right|\left|Y_{\nu 31}\right|$ and $\left|Y_{\nu 22}\right|\left|Y_{\nu 21}\right|$ for the mass spectrum A in Appendix $\mathrm{B}$, corresponding to HENS parameters $m_{H_{u}}^{2}=-(668)^{2} \mathrm{GeV}^{2}, m_{H_{d}}^{2}=$ $-(511)^{2} \mathrm{GeV}^{2}, \tan \beta=10$, and $M_{1 / 2}=300 \mathrm{GeV}$.

the allowed parameter space in this example. However, improved sensitivities from current and future experiments could change this. To illustrate the effects of improved experimental bounds, we also draw a dashed contour in Fig. 9 corresponding to the parameter region that would be allowed with the stronger constraint $B(\tau \rightarrow \mu \gamma)<10^{-10}$. Such a level of sensitivity could potentially be achieved by super $B$ factories [58]. The primary effect of an improvement in the $\tau$ sector bounds is to push the neutrino Yukawa couplings to smaller overall values. Improving $B(\mu \rightarrow e \gamma)$, on the other hand, forces more fine tuning among the different neutrino Yukawa matrix elements.

In Fig. 10 we show the allowed regions in the $\left|Y_{\nu 32}\right|\left|Y_{\nu 31}\right|$ and $M_{N_{1,3}}$ planes for HENS parameter set A points requiring both consistency with the current LFV bounds as well as successful thermal leptogenesis. We have scanned over the neutrino sector parameters in the same way as in Fig. 9. In this plot we also show the regions of the parameter space that would be allowed if the bounds on LFV were improved to $B(\mu \rightarrow e \gamma)<10^{-13}$ and $B(\tau \rightarrow \mu \gamma)<10^{-10}$. As discussed above, strengthening the LFV bounds tends to push the allowed range of $M_{N_{3}}$ to lower values making leptogenesis less effective. 


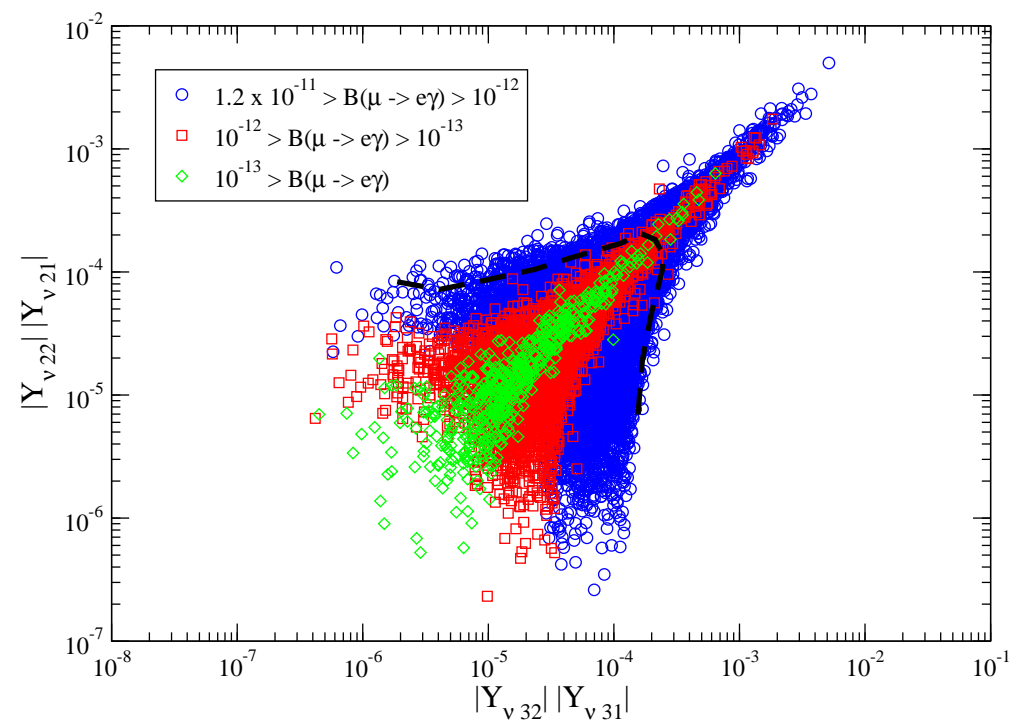

Figure 9: $B(\mu \rightarrow e \gamma)$ in the plane of $\left|Y_{\nu 32}\right|\left|Y_{\nu 31}\right|$ and $\left|Y_{\nu 22}\right|\left|Y_{\nu 21}\right|$ for the mass spectrum A in Appendix B. All points in this figure can account for the baryon asymmetry through thermal leptogenesis. The dashed line corresponds to the region that would still be allowed if the bound on $\tau \rightarrow \mu \gamma$ decay were improved to $B(\tau \rightarrow \mu \gamma)<10^{-10}$.

\section{Conclusions}

We have investigated the consequences of adding right-handed neutrinos to the HENS model. This model provides a simple and phenomenologically consistent solution to the supersymmetric flavor problem. Adding heavy right-handed neutrinos, lepton flavor mixing can arise due to the neutrino Yukawa matrix in the course of RG running. We have studied the constraints on the neutrino-extended HENS model that arise from the current bounds on LFV. We have also investigated whether the baryon asymmetry can be explained by thermal leptogenesis induced by the heavy right-handed neutrinos.

We find that the neutrino-extended HENS model can be consistent with the existing bounds on LFV in two ways. First, the neutrino Yukawa couplings that contribute to lepton flavor mixing can be very small. In the context of a seesaw generating the light neutrino masses, this corresponds to lower values of the right-handed neutrino masses, below about $10^{11} \mathrm{GeV}$. The second way to suppress LFV in the HENS model to arrange for $m_{H_{u}}^{2}$ to be small at the input scale $M_{G U T}$. It is this soft mass that combines with the neutrino Yukawa couplings to source flavor mixing in the RG running. Taking $m_{H_{u}}^{2} \rightarrow 0$ therefore strongly suppresses LFV, even for larger values of the heavy neutrino masses.

In models with heavy right-handed neutrinos, the baryon asymmetry of the universe can be successfully explained by (thermal) leptogenesis. For this mechanism to be effective in the 


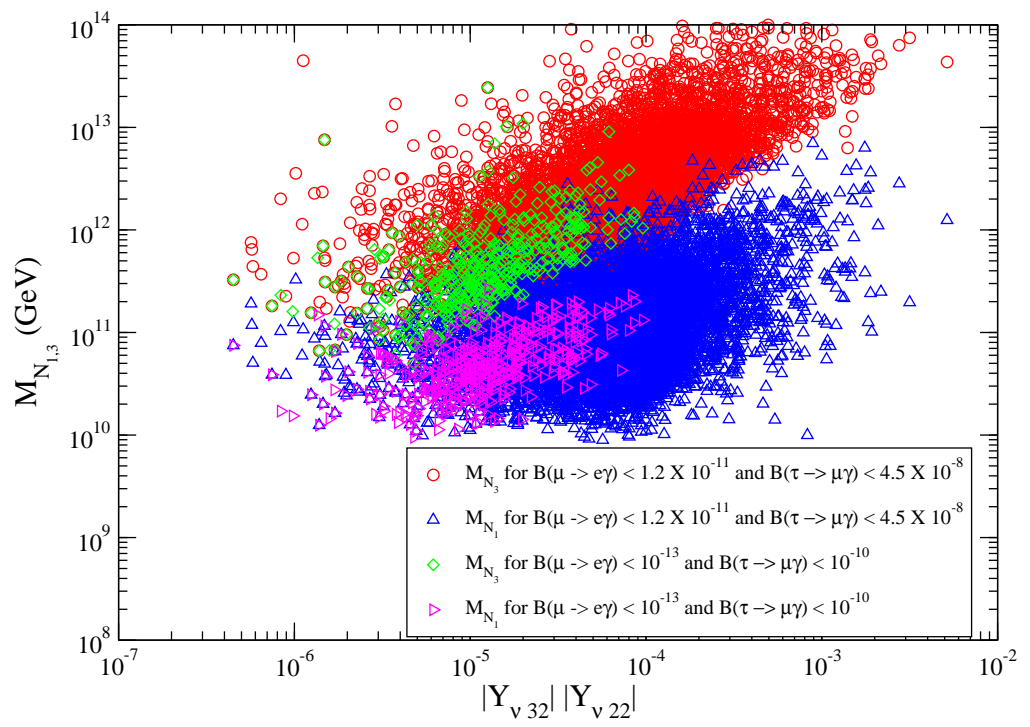

Figure 10: Allowed points subject to the constraints of LFV and thermal leptogenesis for the HENS model parameter set A. The points are plotted as $\left|Y_{\nu 32}\right|\left|Y_{\nu 31}\right|$ against either $M_{N_{1}}$ or $M_{N_{3}}$, with the neutrino sector parameters scanned over.

HENS model, the mass of the lightest right-handed neutrino must exceed about $10^{10} \mathrm{GeV}$. This implies a tension with the constraints from LFV. For both requirements to be met, either $m_{H_{u}}^{2}$ must be somewhat small or the neutrino Yukawa matrix must have a special structure. These constraints will be strengthened by current and upcoming searches for lepton flavor violation.

Our focus has been on enabling a theoretical idea (HENS) to be compatible with additional phenomenological requirements (neutrino masses and small LFV) and explanatory opportunities (baryon asymmetry). Throughout this work, however, it should be noted that even though the HENS idea started out by minimizing LFV in low-scale supersymmetric theories, full compatibility with nature reintroduced flavor violations through neutrino Yukawa effects. This is a generic feature of supersymmetric theories that explicitly incorporate neutrino masses in the spectrum. As explained above, we find LFV bounds nontrivial to satisfy if the baryon asymmetry of the universe originates from thermal leptogenesis with hierarchical right-handed neutrinos. In our view, this highlights in yet another context the importance of making progress in LFV experiments whose non-zero signal upon reaching better sensitivity will be complementary to the knowledge gained from high-energy LHC experiments and will be necessary to unravel the underlying theory. 


\section{Acknowledgements}

We would like to thank Aaron Pierce and Krzysztof Turzynski for helpful conversations. This work was supported by the DOE, the Michigan Center for Theoretical Physics (MCTP), the Korean Institute for Advanced Study (KIAS), and the Kavli Institute for Theoretical Physics (KITP) under the National Science Foundation Grant No. PHY05-51164.

\section{Appendix A: Light Neutrino Parameters}

Neutrino experiments have measured the value of two independent neutrino mass differences: the solar neutrino mass, $\Delta m_{\odot}^{2}$, and the atmospheric neutrino mass, $\Delta m_{@}^{2}$. The $2 \sigma$ ranges of these mass differences are 12

$$
\begin{aligned}
\Delta m_{@}^{2} & =\left|m_{\nu_{3}}^{2}-m_{\nu_{2}}^{2}\right|=(2.1-2.7) \times 10^{-3} \mathrm{eV}^{2} \\
\Delta m_{\odot}^{2} & =m_{\nu_{2}}^{2}-m_{\nu_{1}}^{2}=(7.3-8.1) \times 10^{-5} \mathrm{eV}^{2} .
\end{aligned}
$$

Since the sign of the atmospheric mass difference is undetermined, the hierarchy of the neutrino masses is unknown.

With two known mass differences and three light neutrinos, we can parametrize the masses of all three neutrinos in terms of a single parameter $m_{3}$. In the case of a normal hierarchy $(\mathrm{NH})$, we have

$$
m_{3}=m_{3}, \quad m_{2}=\sqrt{m_{3}^{2}-\Delta m_{@}^{2}}, \quad m_{1}=\sqrt{m_{3}^{2}-\Delta m_{@}^{2}-\Delta m_{\odot}^{2}} .
$$

Demanding that the mass of the lightest right-handed neutrino be real, we obtain a lower bound on the heaviest left-handed neutrino of

$$
m_{3}=\sqrt{\Delta m_{@}^{2}+\Delta m_{\odot}^{2}} \simeq(0.047-0.053) \mathrm{eV} .
$$

We focus on the normal hierarchy in the present work, but we expect our results will be qualitatively the same for an inverted hierarchy (IH).

Whenever we fix a set of low energy neutrino parameters in our analysis, we consider the normal hierarchy with neutrino masses of

$$
m_{1}=9.0 \times 10^{-4} \mathrm{eV}, \quad m_{2}=9.0 \times 10^{-3} \mathrm{eV}, \quad m_{3}=5.0 \times 10^{-2} \mathrm{eV} .
$$

For the mixing angles in the $U$-matrix, defined in Eq. (5), we use the central values of $\theta_{12}$ and $\theta_{23}$, and set $\theta_{13}=0$.

$$
\theta_{12}=35^{\circ}, \quad \theta_{13}=0^{\circ}, \quad \theta_{23}=45^{\circ} \text {. }
$$

These light neutrino parameters are the low-scale values. We do not consider additional RG running of the light neutrino masses. As shown in [59], the RG effects will only make a difference of $10-15 \%$. This will not qualitatively alter our results. 


\section{Appendix B: Sample Mass Spectrum}

We list in Table 1 the high-scale input HENS model parameters for points A and B discussed in the text. We also list some of the relevant low-scale model parameters obtained by RG running using SuSpect 2.34 [60]. In Table 2 we collect the relevant superpartner masses corresponding to points A and B. Again, these were obtained using SuSpect 2.34 [60].

\begin{tabular}{|c|c||c|}
\hline Parameter & $\mathrm{A}(\mathrm{GeV})$ & $\mathrm{B}(\mathrm{GeV})$ \\
\hline \hline$M_{1 / 2}$ & 300 & 300 \\
$\tan \beta$ & 10 & 10 \\
$\operatorname{Sgn} \operatorname{Sqrt}\left(m_{H_{u}}^{2}\right)$ & -668 & -100 \\
$\operatorname{Sgn} \operatorname{Sqrt}\left(m_{H_{d}}^{2}\right)$ & -511 & -359 \\
$\operatorname{sgn}(\mu)$ & + & + \\
\hline \hline$M_{1}$ & 123 & 122 \\
$M_{2}$ & 231 & 230 \\
$\mu$ & 666 & 401 \\
$M_{A^{0}}$ & 851 & 566 \\
$m_{\tilde{L}_{1,2}}^{2}$ & 148 & 192 \\
$m_{\tilde{E}_{1,2}}^{2}$ & 221 & 140 \\
\hline
\end{tabular}

Table 1: High-scale HENS model input parameters and selected low-scale output parameters for the sample points A and B discussed in the text.

\begin{tabular}{|c|c|c||c|c|c|}
\hline Particle & $\mathrm{A}(\mathrm{GeV})$ & $\mathrm{B}(\mathrm{GeV})$ & Particle & $\mathrm{A}(\mathrm{GeV})$ & $\mathrm{B}(\mathrm{GeV})$ \\
\hline \hline$m_{\chi_{1}^{0}}$ & 120 & 118 & $m_{\tilde{\nu}_{e}}$ & 134 & 180 \\
$m_{\chi_{2}^{0}}$ & 230 & 219 & $m_{\tilde{e}_{L}}$ & 155 & 197 \\
$m_{\chi_{3}^{0}}$ & 667 & 407 & $m_{\tilde{e}_{R}}$ & 225 & 146 \\
$m_{\chi_{4}^{0}}$ & 673 & 425 & $m_{\tilde{\nu}_{\tau}}$ & 131 & 179 \\
$m_{\chi_{1}^{ \pm}}$ & 230 & 219 & $m_{\tilde{\tau}_{1}}$ & 136 & 132 \\
$m_{\chi_{2}^{ \pm}}$ & 674 & 425 & $m_{\tilde{\tau}_{2}}$ & 231 & 201 \\
\hline
\end{tabular}

Table 2: Low-scale superpartner masses for the sample points A and B discussed in the text. 


\section{References}

[1] J. S. Hagelin, S. Kelley and T. Tanaka, Nucl. Phys. B 415, 293 (1994).

[2] F. Gabbiani, E. Gabrielli, A. Masiero and L. Silvestrini, Nucl. Phys. B 477, 321 (1996) hep-ph/9604387.

[3] J. R. Ellis, C. Kounnas and D. V. Nanopoulos, Nucl. Phys. B 247, 373 (1984).

[4] D. E. Kaplan, G. D. Kribs and M. Schmaltz, Phys. Rev. D 62, 035010 (2000) hep$\mathrm{ph} / 9911293$.

[5] Z. Chacko, M. A. Luty, A. E. Nelson and E. Ponton, JHEP 0001, 003 (2000) hepph/9911323.

[6] M. Schmaltz and W. Skiba, Phys. Rev. D 62, 095005 (2000) |hep-ph/0001172]; M. Schmaltz and W. Skiba, Phys. Rev. D 62, 095004 (2000) [hep-ph/0004210].

[7] A. E. Nelson and M. J. Strassler, JHEP 0009, 030 (2000) hep-ph/0006251; A. E. Nelson and M. J. Strassler, JHEP 0207, 021 (2002) [hep-ph/0104051].

[8] J. L. Evans, D. E. Morrissey and J. D. Wells, Phys. Rev. D 75, 055017 (2007) hep$\mathrm{ph} / 0611185$.

[9] M. A. Luty and R. Sundrum, Phys. Rev. D 65, 066004 (2002) hep-th/0105137; M. Luty and R. Sundrum, Phys. Rev. D 67, 045007 (2003) [hep-th/0111231].

[10] T. S. Roy and M. Schmaltz, arXiv:0708.3593 [hep-ph].

[11] H. Murayama, Y. Nomura and D. Poland, Phys. Rev. D 77, 015005 (2008) arXiv:0709.0775 [hep-ph]].

[12] For recent reviews of neutrino physics, see:

R. N. Mohapatra et al., Rept. Prog. Phys. 70, 1757 (2007) hep-ph/0510213]; R. N. Mohapatra and A. Y. Smirnov, Ann. Rev. Nucl. Part. Sci. 56, 569 (2006) [hep-ph/0603118];

A. Strumia and F. Vissani, [hep-ph/0606054].

[13] F. Borzumati and A. Masiero, Phys. Rev. Lett. 57, 961 (1986).

[14] M. Fukugita and T. Yanagida, Phys. Lett. B 174, 45 (1986).

[15] M. A. Luty, Phys. Rev. D 45, 455 (1992).

[16] F. R. Klinkhamer and N. S. Manton, Phys. Rev. D 30, 2212 (1984).

[17] V. A. Kuzmin, V. A. Rubakov and M. E. Shaposhnikov, Phys. Lett. B 155, 36 (1985).

[18] A. D. Sakharov, Pisma Zh. Eksp. Teor. Fiz. 5, 32 (1967) [JETP Lett. 5, 24 (1967 SOPUA,34,392-393.1991 UFNAA,161,61-64.1991)]. 
[19] S. Pascoli, S. T. Petcov and C. E. Yaguna, Phys. Lett. B 564, 241 (2003) hepph/0301095]; S. Pascoli, S. T. Petcov and W. Rodejohann, Phys. Rev. D 68, 093007 (2003) [hep-ph/0302054].

[20] P. H. Chankowski, J. R. Ellis, S. Pokorski, M. Raidal and K. Turzynski, Nucl. Phys. B 690297 (2004) hep-ph/0403180.

[21] S. T. Petcov, W. Rodejohann, T. Shindou and Y. Takanishi, Nucl. Phys. B 739, 208 (2006) [hep-ph/0510404.

[22] S. Antusch, E. Arganda, M. J. Herrero and A. M. Teixeira, JHEP 0611, 090 (2006) hep-ph/0607263.

[23] M. Maltoni, T. Schwetz, M. A. Tortola and J. W. F. Valle, New J. Phys. 6, 122 (2004) hep-ph/0405172.

[24] G. L. Fogli, E. Lisi, A. Marrone and A. Palazzo, Prog. Part. Nucl. Phys. 57, 742 (2006) hep-ph/0506083.

[25] B. Pontecorvo, Sov. Phys. JETP 6, 429 (1957) [Zh. Eksp. Teor. Fiz. 33, 549 (1957)].

[26] Z. Maki, M. Nakagawa and S. Sakata, Prog. Theor. Phys. 28, 870 (1962).

[27] J. A. Casas and A. Ibarra, Nucl. Phys. B 618, 171 (2001) hep-ph/0103065.

[28] S. T. Petcov, S. Profumo, Y. Takanishi and C. E. Yaguna, Nucl. Phys. B 676, 453 (2004) [hep-ph/0306195].

[29] J. Hisano, T. Moroi, K. Tobe, M. Yamaguchi and T. Yanagida, Phys. Lett. B 357, 579 (1995) [hep-ph/9501407]; J. Hisano, T. Moroi, K. Tobe and M. Yamaguchi, Phys. Rev. D 53, 2442 (1996) [hep-ph/9510309].

[30] J. Hisano and D. Nomura, Phys. Rev. D 59, 116005 (1999) hep-ph/9810479].

[31] I. Masina and C. A. Savoy, Nucl. Phys. B 661, 365 (2003) hep-ph/0211283.

[32] J. L. Feng, A. Rajaraman and B. T. Smith, Phys. Rev. D 74, 015013 (2006) hepph/0512172.

[33] R. R. de Austri, R. Trotta and L. Roszkowski, JHEP 0605, 002 (2006) [hep-ph/0602028]; B. C. Allanach, Phys. Lett. B 635, 123 (2006) [hep-ph/0601089]; B. C. Allanach, C. G. Lester and A. M. Weber, JHEP 0612, 065 (2006) [hep-ph/0609295]; B. C. Allanach, K. Cranmer, C. G. Lester and A. M. Weber, JHEP 0708, 023 (2007) [0705.0487 [hep-ph]].

[34] M. L. Brooks et al. [MEGA Collaboration], Phys. Rev. Lett. 83, 1521 (1999) hepex/9905013.

[35] B. Aubert et al. [BABAR Collaboration], Phys. Rev. Lett. 95, 041802 (2005) hepex/0502032. 
[36] K. Hayasaka et al. [Belle Collaboration], 0705.0650 [hep-ex].

[37] B. Aubert et al. [BABAR Collaboration], Phys. Rev. Lett. 96, 041801 (2006) hepex/0508012.

[38] E. Komatsu et al. [WMAP Collaboration], arXiv:0803.0547] [astro-ph].

[39] A. Abada, S. Davidson, A. Ibarra, F. X. Josse-Michaux, M. Losada and A. Riotto, JHEP 0609, 010 (2006) hep-ph/0605281.

[40] A. Abada, S. Davidson, F. X. Josse-Michaux, M. Losada and A. Riotto, JCAP 0604, 004 (2006) hep-ph/0601083.

[41] E. Nardi, Y. Nir, J. Racker and E. Roulet, JHEP 0601, 068 (2006) hep-ph/0512052].

[42] E. Nardi, Y. Nir, E. Roulet and J. Racker, JHEP 0601, 164 (2006) hep-ph/0601084.

[43] E. Nardi, J. Racker and E. Roulet, JHEP 0709, 090 (2007) [0707.0378 [hep-ph]].

[44] S. Davidson and A. Ibarra, Phys. Lett. B 535, 25 (2002) hep-ph/0202239.

[45] G. F. Giudice, A. Notari, M. Raidal, A. Riotto and A. Strumia, Nucl. Phys. B 685, 89 (2004) hep-ph/0310123.

[46] T. Hambye, Y. Lin, A. Notari, M. Papucci and A. Strumia, Nucl. Phys. B 695, 169 (2004) hep-ph/0312203.

[47] M. Bolz, A. Brandenburg and W. Buchmuller, Nucl. Phys. B 606, 518 (2001) [Erratumibid. B 790, 336 (2008)] [hep-ph/0012052].

[48] W. Buchmuller, K. Hamaguchi and J. Kersten, Phys. Lett. B 632, 366 (2006) hepph/0506105]. W. Buchmuller, J. Kersten and K. Schmidt-Hoberg, JHEP 0602, 069 (2006) [hep-ph/0512152]. W. Buchmuller, L. Covi, J. Kersten and K. Schmidt-Hoberg, JCAP 0611, 007 (2006) hep-ph/0609142.

[49] M. Kawasaki, K. Kohri and T. Moroi, Phys. Rev. D 71, 083502 (2005) astroph/0408426].

[50] M. Flanz, E. A. Paschos and U. Sarkar, Phys. Lett. B 345, 248 (1995) [Erratum-ibid. B 382, 447 (1996)] |hep-ph/9411366]; M. Flanz, E. A. Paschos, U. Sarkar and J. Weiss, Phys. Lett. B 389, 693 (1996) [hep-ph/9607310].

[51] L. Covi, E. Roulet and F. Vissani, Phys. Lett. B 384, 169 (1996) hep-ph/9605319.

[52] A. Pilaftsis, Phys. Rev. D 56, 5431 (1997) hep-ph/9707235.

[53] A. Pilaftsis and T. E. J. Underwood, Nucl. Phys. B 692, 303 (2004) hep-ph/0309342.

[54] T. Hambye, J. March-Russell and S. M. West, JHEP 0407, 070 (2004) arXiv:hepph/0403183. 
[55] T. Asaka, K. Hamaguchi, M. Kawasaki and T. Yanagida, Phys. Lett. B 464, 12 (1999) hep-ph/9906366]; T. Asaka, K. Hamaguchi, M. Kawasaki and T. Yanagida, Phys. Rev. D 61, 083512 (2000) hep-ph/9907559].

[56] G. F. Giudice, M. Peloso, A. Riotto and I. Tkachev, JHEP 9908, 014 (1999) hepph/9905242]; G. F. Giudice, A. Riotto and A. Zaffaroni, Nucl. Phys. B 710, 511 (2005) [hep-ph/0408155]; G. F. Giudice, L. Mether, A. Riotto and F. Riva, 0804.0166 [hep-ph].

[57] T. Mori, Nucl. Phys. Proc. Suppl. 169, 166 (2007).

[58] J. L. . Hewett et al., hep-ph/0503261.

[59] S. Antusch, J. Kersten, M. Lindner and M. Ratz, Nucl. Phys. B 674, 401 (2003) hepph/0305273.

[60] A. Djouadi, J. L. Kneur and G. Moultaka, Comput. Phys. Commun. 176, 426 (2007) hep-ph/0211331. 